\title{
亶 \\ Importância da antissepsia cirúrgica na prevenção de infecção no pós-operatório em pequenos animais
}

[Importance of surgical antisepsis in the prevention of infection in the postoperative period in small animals]

\section{"Revisão/Review"}

\section{Sabrina Cândido Trajano*, Breno Bezerra Aragão, Mauro Araujo Penaforte Junior, Raylson Pereira Oliveira, Gabriela Ratis Galeas, Karine Silva Camargo, Maria Sheila Silva Ferreira, Grazielle Anahy Sousa Aleixo}

${ }^{1}$ Departamento de Medicina Veterinária, Universidade Federal Rural de Pernambuco, Recife-PE, Brasil.

*Autor para correspondência/Corresponding author: E-mail: sabrinacandidotrajano@ gmail.com

\section{Resumo}

Sabe-se que toda intervenção cirúrgica está sujeita à ocorrência de processos infecciosos e que a pele do paciente é uma das fontes de infecção, tornando, portanto, a antissepsia do campo operatório de cães e gatos um procedimento indispensável. A infecção do sítio cirúrgico (ISC) pode se manifestar em até um ano após a realização do procedimento do cirúrgico, sendo uma das principais causas de morbidade e mortalidade de pacientes no pós-operatório. A antissepsia é definida como o ato de eliminar micro-organismos existentes nas camadas superficiais e profundas da pele, mediante aplicação de um agente germicida hipoalergênico, de baixa causticidade passível de ser aplicado em tecido vivo. Diversas soluções antissépticas podem ser utilizadas na antissepsia cutânea. Os produtos mais utilizados são o álcool a $70 \%$, iodopovidona $10 \%$ e o gluconato de clorexidina alcoólica $0,5 \%$ e a $2 \%$ que podem ser encontrados em formulações aquosa, alcoólica e degermante. Diante das informações descritas e tendo em vista a importância da prevenção de infecções cirúrgicas na medicina veterinária objetiva-se com este trabalho fazer um levantamento bibliográfico sobre a antissepsia como medida profilática de infecções no pós-operatório de pequenos animais.

Palavras-chave: assepsia; campo operatório; evolução da cirurgia; medida preventiva.

\begin{abstract}
It is known that any surgical intervention is subject to the occurrence of infectious processes and the patient's skin is one of the sources of infection, thus making antisepsis of the operative field of dogs and cats an indispensable procedure. Surgical site infection (SSI) can manifest itself within one year after the surgical procedure, being one of the main causes of postoperative morbidity and mortality in patients. Antisepsis is defined as the act of eliminating existing microorganisms in the superficial and deep layers of the skin by application of a hypoallergenic germicidal agent of low causticity capable of being applied in living tissue. Several antiseptic solutions can be used in cutaneous antisepsis. The most commonly used products are $70 \%$ alcohol, $10 \%$ iodopovidone and $0.5 \%$ and $2 \%$ alcohol chlorhexidine gluconate which can be found in aqueous, alcoholic and degerming solutions. In view of the information described and considering the importance of preventing surgical infections in veterinary medicine, this paper aims to make a bibliographic survey on antisepsis as a prophylactic measure of infections in the postoperative period of small animals.
\end{abstract}

Keywords: asepsis; operative field; evolution of surgery; preventive measure. 


\section{Introdução}

$\mathrm{Na}$ veterinária, as infecções hospitalares podem causar graves consequências ao paciente, principalmente ao seu bem-estar e ainda resultar em danos psicológicos e financeiros ao tutor, além de contribuir para grandes perdas econômicas quando ocorre em animais de produção. Por isso deve-se buscar a prevenção de infecções, o que exige precauções com relação à antissepsia do paciente e da equipe cirúrgica, apesar de muitos profissionais na medicina veterinária negligenciarem tais medidas de controle (Stehling et al., 2001).

Apesar dos avanços nas práticas de controle de infecções hospitalares alcançados nos últimos anos, as infecções do sítio cirúrgico (ISC) ainda estão entre as principais causas de morbidade e mortalidade de pacientes humanos e animais no período pós-operatório (Weese, 2008; Singh e Weese, 2017), podendo se manifestar em até 30 dias de pós-operatório, porém em casos em que se utilizam implantes ou prótese pode ocorrer até um ano após a cirurgia (Poveda et al., 2003).

Ao longo dos anos, diversas soluções antissépticas foram desenvolvidas, todas com o objetivo de evitar ou diminuir o risco de infecções (Phillips et al., 1991). Os produtos mais utilizados na antissepsia cutânea dos animais são o álcool a $70 \%$, iodopovidona $10 \%$ (PVPI) e o gluconato de clorexidina $(\mathrm{CHG})$, este pode ser encontrado em várias concentrações e em formulações aquosa, alcoólica e degermante (Moriya e Módena, 2008).

Embora a utilização dos antissépticos na preparação da pele dos pacientes estar bem difundida na medicina veterinária, ainda não se sabe qual a solução que possui melhor eficácia (Garcia, 2010), apesar de alguns trabalhos demonstrarem que o $\mathrm{CHG}$ tem melhor ação bactericida na antissepsia de cães e gatos (Boucher et al., 2017; Privitera et al., 2017).

Devido à importância da prevenção de infecções cirúrgicas na medicina veterinária, este trabalho tem como objetivo fazer uma revisão literária sobre a importância da antissepsia cirúrgica como medida profilática de infecções no pós-operatório em pequenos animais.

\section{Desenvolvimento}

Infecções no sítio cirúrgico

A infecção do sítio cirúrgico (ISC) é aquela que ocorre na ferida cirúrgica, órgãos, tecidos ou em cavidades que foram manipuladas durante um procedimento cirúrgico (Dunning, 2007). A ISC pode ser classificada em superficial quando envolve apenas a pele e/ou tecido subcutâneo do local da incisão associado a secreção purulenta, calor, dor e edema localizado na região. Pode ser profunda, quando invade tecidos mais profundos como a fáscia e musculatura, apresenta secreção purulenta, deiscência da ferida cirúrgica, febre, dor, cultura positiva para bactérias e abscesso e pode ainda envolver órgãos e outras estruturas manipuladas durante o procedimento. Esta classificação pode ser igualmente aplicada na medicina veterinária (Weese, 2008; Singh e Weese, 2017).

Com relação ao paciente, a ISC causa dor, desconforto, estresse e piora sua qualidade de vida, além de aumentar os retornos ao hospital veterinário, com gastos não planejados para a resolução do problema. Pode ainda levar a complicações graves no pós-cirúrgico, como osteomielite, bacteremia, choque séptico e morte dos animais (Seliskar et al., 2007).

Apesar de poucos estudos alguns fatores tem sido associados a ISC na medicina veterinária. Entre eles, o número de animais hospitalizados, uso indiscriminado de antimicrobianos, emprego de técnicas de antissepsia inadequadas, tricotomia realizada mais de duas horas antes da cirurgia, o tipo do procedimento cirúrgico, número de microorganismos presentes no tecido a ser operado antes do procedimento cirúrgico (Dunning, 2007), idade do paciente, doença prévia, desnutrição, tipo e duração do procedimento cirúrgico, uso de implantes ortopédicos (Mangram et al., 1999), instrumental, vestimenta (Harari, 2004) e classificação do risco anestésico (Fernandes, 2000).

$\mathrm{Na}$ medicina humana, há vários estudos associando a ISC com os micro-organismos presentes no campo operatório, enquanto que na medicina veterinária há poucos dados correlacionando estes fatores (Fernando et al., 2015). No entanto a taxa de ISC nos animais é semelhante à encontrada em humanos, a qual pode variar de $0,8 \%$ quando se fala em qualquer tipo de procedimento cirúrgico, de $3,6 \%$ a $15,8 \%$ para cirurgias quando há uso de implante ortopédico e $18,1 \%$ para cirurgias consideradas contaminadas (Singh e Weese, 2017).

Em estudo realizado por Corsini et al. (2014) no Hospital Veterinário de Viçosa foi demonstrado uma taxa de $24,5 \%$ de ISC. Em Illinois, nos 
Estados Unidos, dos 7.965 procedimentos ortopédicos realizados em cães, gatos e equinos, $4,32 \%$ dos animais (344) apresentaram ISC (Weese, 2008). Outros autores relatam uma taxa de 5,5 a 9,5\% de ISC no pós-cirúrgico de pequenos animais (Viana, 2001; Dunning, 2007; Arias et al., 2013).

Apesar de poucos estudos, sabe-se que a ISC está relacionada diretamente a microbiota cutânea dos cães e gatos, sendo esta formada por dois tipos de micro-organismos, conhecidos como transitórios e os residentes. A primeira se refere aos micro-organismos ambientais recentes, que são considerados não colonizantes e sobrevivem por curto período de tempo na pele, como as bactérias Gram-negativos, entre elas a Escherichia coli. As bactérias residentes apresentam maior resistência e possuem maior período de vida na pele, e por isso são considerados colonizantes, como Staphylococcus spp. e Corynebacterium spp. A pele do animal é a fonte mais comum de infecção, sendo as bactérias do gênero Staphylococcus as de maior importância na clínica médica e cirúrgica de pequenos animais (Murray, 1995).

O conhecimento das espécies de microorganismos presentes no campo operatório dos animais assim como a sensibilidade dos mesmos frente aos antimicrobianos é de grande importância na prevenção de ISC (Fernando et al., 2015). Algumas infecções generalizadas foram associadas à contaminação pelo Acinetobacter baumannii, sendo este um micro-organismo de importância médica veterinária (Boerlin et al., 2001; Francey et al., 2008).

\section{História da antissepsia}

Até a metade do século XIX as infecções cirúrgicas foram um grande empecilho para a evolução e desenvolvimento da cirurgia. Foi a partir da descoberta de métodos de antissepsia em meados do século XIX que a cirurgia passou por uma grande evolução (Dellinger, 2003). Antes das descobertas dos micro-organismos, da antissepsia e sua importância na infecção, os procedimentos cirúrgicos eram realizados de forma cruenta e sem perspectiva de sobrevivência do paciente sendo os mesmos realizados pelos chamados barbeiros cirurgiões (Amato, 2006).

Em 1843, Oliver Wendell Holmes sugeriu que a febre puerperal era transmitida pelos obstetras de uma pessoa a outra, mas sua teoria não foi aceita. Posteriormente em 1847, o médico húngaro Ignaz Philipp Semmelweis, demostrou a origem da infecção da febre puerperal comprovando que ela era transmitida pelos próprios médicos quando eles atendiam as pacientes grávidas após realizarem autópsia de outras pacientes que haviam morrido pela doença, o que reforçou a teoria de Holmes. A mortalidade na enfermaria das parteiras chegava a $3 \%$, enquanto que na enfermaria dos médicos chegava a $20 \%$. A partir de então, passou-se a instituir a higiene com hipoclorito de cálcio na lavagem das mãos da equipe médica antes de entrar nas salas da clínica obstétrica, reduzindo assim a taxa de mortalidade (Amato, 2006; Fernandes et al., 2005).

Durante a guerra da Criméria no ano de 1854, Florence Nightingale juntamente com outras enfermeiras passaram a usar medidas de higiene com os soldados feridos em campo de batalha, melhorando as condições de tratamento nos hospitais improvisados. (Carraro, 2004; Stanley, 2007).

Em 1860, Joseph Lister (1867) observou que as supurações (infecção) das feridas eram provocadas por influência atmosférica sobre o sangue e soro que ficavam nos tecidos lesionados. Baseado nas descobertas de Pasteur percebeu que a infecção causada pela atmosfera poderia ser evitada aplicando curativo com algum produto capaz de destruir a vida dos 'germes' do ar. Em seus experimentos ele passou a utilizar compressas banhadas em ácido fênico (cobertas com uma tampa para evitar que o produto evaporasse) nas feridas cirúrgicas e assim conseguiu manter as feridas livres de contaminação microbiana em todos os pacientes (Lister, 1867; Amato, 2006; Fontana, 2006; Rutkow, 2013). Posteriormente ele criou uma pasta com o produto para cobrir feridas contaminadas e tratar fraturas expostas. Também pulverizava o produto nas enfermarias, mantendo assim os procedimentos cirúrgicos livres de infecções. Em sua enfermaria nenhum caso de septicemia, erisipela ou gangrena hospitalar' ocorreram após o uso do antisséptico, mesmo essas afecções sendo as principais causas de morte nos hospitais da época (Lister, 1867; Lister, 1870; Rutkow, 2013).

De acordo com Lister (1867), qualquer sinal de infecção após a cirurgia é indicação de o que a assepsia falhou. Na época os cirurgiões ingleses se opuseram às suas ideias, no entanto, elas se espalharam pelo continente europeu sendo seguida pelos cirurgiões Alemães e Austríacos (Rezende, 2009). Cirurgiões alemães perceberam a importância do fenol na etiologia das infecções, 
reconhecendo assim a importância da antissepsia na prática médica (Amato, 2006). Mesmo diluindo o fenol, a substância era tóxica e muitos se recusavam a usar, mas em 1878, ele já era empregado no mundo todo. A primeira cirurgia asséptica só foi possível com o uso do fenol, entretanto atualmente o fenol é raramente usado como antisséptico (Couteur e Burreson, 2009).

Além dos métodos de antissepsia também foi descoberto a importância de esterilizar os materiais utilizados nas cirurgias, como a primeira autoclave criada em 1879 pelo pupilo de Pasteur, Charles Chamberland (Santiago, 2005), e também o uso de uma 'blusa' especialmente para ser vestida na sala de cirurgia instituída por Daniel Mollière e o uso de luvas de borracha introduzido por Willian Halsted. As máscaras, tocas e aventais foram introduzidas depois, bem como a cobertura de mesas e dos pacientes com campos operatórios. Com o passar do tempo o centro cirúrgico passou a ser um ambiente limpo e asséptico diminuindo consideravelmente os casos de ISC (Amato, 2006).

\section{Antissepsia}

A antissepsia é o ato de eliminar microorganismos existentes nas camadas superficiais e profundas da pele, mediante aplicação de um agente germicida hipoalergênico, de baixa causticidade e passível de ser aplicado em um tecido vivo (Moriya e Módena, 2008; Reis, 2011). A antissepsia cutânea e das mãos da equipe cirúrgica reduz de forma significativa os riscos de infecções no pós-operatório do paciente (Orberg et al., 2007; Felix e Miyadahira, 2009), representando um papel essencial na realização de um procedimento cirúrgico (Monteiro et al., 2001; Magalini et al., 2012).

Devido à presença de pelos e a pouca frequência de banhos, a preparação da pele dos animais é um procedimento desafiador na medicina veterinária (Bhavan e Warren, 2009). Para tal procedimento, existem diversos protocolos para antissepsia do paciente, bem como diferentes soluções antissépticas podem ser utilizadas sozinhas ou associadas, e podem ou não ser aplicadas com friç̧ão, de forma que também permita a remoção de micro-organismos de forma mecânica (Cheadle, 2006; Goulart et al., 2011).

Medeiros et al. (2018) relatam que o uso de clorexedina $0,5 \%$ apresentou melhor ação na antissepsia de cadelas submetidas à ovariohisterectomia eletiva quando comparado ao uso de polivinilpirrolidona.
Falha na antissepsia está entre as principais causas de ISC (McNeil et al., 2001; Cheadle, 2006; Corsini et al., 2014). Mesmo antes da realização do procedimento cirúrgico, os micro-organismos causadores de infecção da ferida cirúrgica já estão presentes no campo operatório, por isso a importância de se realizar uma antissepsia eficiente (Levin e Dias, 2006).

\section{Soluções antissépticas}

As soluções antissépticas são agentes biocidas capazes de eliminar ou inibir a proliferação de micro-organismo presentes na pele e na mucosa (Reis, 2011; Pelosi, 2017). O antisséptico deve eliminar totalmente os microorganismos transitórios da pele e reduzir de forma significativa a microbiota residente e inibir seu crescimento até o término do procedimento cirúrgico (Moriya e Módena, 2008; WHO, 2009).

Antes do uso da solução antisséptica a pele do paciente deve ser preparada realizando tricotomia ampla para retirada dos pelos e lavagem prévia para remover sujidades e oleosidade, tendo o cuidado para não causar micro lesões na pele. Para a antissepsia podem ser utilizados soluções a base de iodóforo, clorexidina, álcool, hexaclorofeno e sais quartenários de amônia (Schulz, 2014). Os mais utilizados atualmente na medicina veterinária são o CHG, o PVPI, o álcool a $70 \%$ e o triclosan (Gonçalves et al., 2012; Pelosi, 2017).

O antisséptico cirúrgico ideal deve ser ativo contra uma grande variedade de micro-organismos, promovendo a morte rápida de formas vegetativas e esporuladas, deve estar disponível em soluções detergentes que removam sujidades e oleosidade da pele, ser de fácil aplicação, não causar reação inflamatória na pele, sendo capaz de manter sua atividade na presença de matéria orgânica e ter efeito residual prolongado. As soluções antissépticas podem ser aplicadas a pele do paciente através da técnica do esfregaço, spray ou simplesmente derramada no campo operatório deixando agir pelo tempo desejado (Phillips et al., 1991; Schulz, 2014; Pelosi, 2017).

Álcool 70\%

$\mathrm{O}$ álcool $\left(\mathrm{C}_{2} \mathrm{H}_{6} \mathrm{O}\right)$ está disponível em concentrações que variam de 60 a $80 \%$ apresentam ação bactericida imediata com ação efetiva em 15 segundos, no entanto algumas de suas características limitam o seu uso, como sua capacidade volátil e rápida evaporação em 
temperatura ambiente, por ser altamente inflamável, por causar o ressecamento da pele devido à remoção da gordura quando utilizado com frequência e sem adição de emoliente, como a glicerina a $2 \%$ e redução da capacidade bactericida na presença de matéria orgânica (Santos et al., 2002). Quando associado a algum produto emoliente, sua atividade antimicrobiana é aumentada devido a menor capacidade de evaporação do álcool (Santos et al., 2002). Não é recomendado seu uso para remover sujidades da pele devido o ressecamento que pode provocar (Burg et al., 2007). É indicado apenas para a higienização das mãos, coto umbilical e antissepsia da pele para punção venosa ou arterial (Anvisa, 2010; Anvisa, 2016).

$\mathrm{O}$ produto age rompendo as membranas e provocando rápida desnaturação proteica e consequente lise das bactérias Gram-positivos e Gram-negativos, principalmente contra o bacilo da tuberculose, além de ser fungicida e ter ação sobre alguns tipos de vírus incluindo o vírus da hepatite B e HIV (Vírus da imunodeficiência humana) (Anvisa, 2010; Anvisa, 2016). Soluções alcoólicas em concentrações acima de $80 \%$ são menos efetivas do que concentrações mais baixas (60$80 \%$ ), devido a dificuldade de desnaturação das proteínas na ausência de água (Anvisa, 2010). De acordo com Santos (2002) produtos à base de álcool etílico são mais eficientes para antissepsia. Como desvantagem não exerce ação residual (Moriya e Módena, 2008) e não apresenta eficiência contra esporos, e por isso deve ser utilizar como preparação alcoólica (PA) em associação com CHG ou PVPI (Moriya e Módena, 2008; Gonçalves et al, 2012).

\section{Gluconato de Clorexidina}

$\mathrm{O}$ CHG $\left(\mathrm{C}_{22} \mathrm{H}_{30} \mathrm{Cl}_{2} \mathrm{~N}_{10}\right)$ foi inicialmente utilizado na Inglaterra em 1954 e foi difundido mundialmente como produto antisséptico tópico e desinfetante (Milstone et al., 2008). Na odontologia, seu emprego começou a partir da década de 1970, época em que foi demonstrada sua capacidade em reduzir a formação de biofilmes bacterianos e a gengivite (Sievert et al., 2011).

Esse antisséptico pertence à classe das biguanidas e tem como característica uma base forte com propriedades catiônicas, o que possibilita se ligar à parede celular do micro-organismo alterando o equilíbrio osmótico, resultando na ruptura de suas membranas celulares. Quando aplicada em baixas concentrações, consegue atravessar a parede celular do micro-organismo por difusão passiva, prejudicando a permeabilidade da membrana e resultando em extravasamento de material citoplasmático. Quando utilizada em altas concentrações, causa a coagulação de constituintes intracelulares. Na presença de matéria orgânica como o sangue, sua ação não é inativada (Mcdonnell e Russel, 1999).

As soluções antissépticas podem causar reação cutânea imediata, no entanto, o CHG acarreta menos dermatites de contato que outras soluções antissépticas (ANVISA, 2010).

Atualmente, a clorexidina está disponível em diversas concentrações, variando de $0,2 \%$ a $4 \%$, e em diferentes formulações, podendo ou não conter álcool isopropil ou etanol. Para fins odontológicos também pode ser veiculada na forma de enxaguante bucal, aerossóis, gel e verniz (Moriya e Módena, 2008).

A clorexidina apresenta início de ação efetiva em 15 segundos reduzindo rapidamente a flora bacteriana transitória da pele e o seu efeito residual impede o crescimento rápido dos microorganismos por até seis horas, principalmente quando associado ao álcool. Tem melhor atividade contra bactérias Gram-positivos apesar de também ter ação contra bactérias Gram-negativos, aeróbios e anaeróbios facultativos, fungos e alguns vírus como o HIV. É efetiva mesmo na presença de fluidos corporais, no entanto não é esporicida (Da Cunha et al., 2008; Moriya e Módena, 2008).

A solução alcoólica de clorexidina alcoólico $0,5 \%$ é indicada para a antissepsia complementar da pele do sítio cirúrgico, para acesso venoso e arterial, e também para a realização de procedimentos invasivos como passagem de cateter venoso central, toracocentese, biópsias, abdominocentese entre outros (Anvisa, 2010; Anvisa, 2016).

O CHG $0,5 \%$ a $2 \%$ é indicado para higiene das mãos e antissepsia da pele do campo operatório, quando preparado na forma de degermante ou alcóolico. Tem sido utilizada para controlar surtos de Staphylococcus aureus, inclusive os que apresentam resistência a meticilina em unidades intensivas de tratamento em hospitais humanos, através de banhos diários e do uso intranasal da clorexidina associada a mupirocina em adultos e crianças recém-nascidas (Casey e Lambert, 2007; Da Cunha et al., 2008; Anvisa, 2010; Sievert et al., 2011; Casey et al., 2015; Anvisa, 2016). 


\section{Iodopovidona}

O iodo é um antisséptico reconhecido pela sua efetividade desde 1821 , no entanto devido as propriedades de causar irritação e de manchar a pele ele foi substituído pelo PVPI em 1960 (Anvisa, 2010). Em 1953, Shelanski e Shelanski descobriram que o iodo poderia ser diluído em polivinilpirrolidona ( $\mathrm{PVP}, \mathrm{C}_{6} \mathrm{H}_{9} \mathrm{NO}$ ), um polímero usado para prolongar a atividade farmacológica de medicamentos. Os iodóforos são moléculas compostas de iodo e de um polímero carreador (PVP) que liberam o iodo gradativamente permitindo maior estabilidade e solubilidade para o produto, com maior tempo de ação residual, menor irritabilidade cutânea e não causa manchas na pele (Da Cunha et al., 2008; Brandão, et al., 2015).

O iodo é um halogênico, que pode ser encontrado em formulações aquosas, alcoólicas e degermante. A quantidade de iodo livre na molécula é que determina o grau de efetividade antimicrobiana do produto, sendo o PVPI a $10 \%$ contendo $1 \%$ de iodo livre dissolvido em solução alcoólica o mais utilizado na antissepsia cutânea (Anvisa, 2010).

O PVPI penetra na parede celular, inativando as células devido a formação de complexos com aminoácidos e ácidos graxos insaturados, prejudicando a síntese proteica e alterando as membranas celulares (Anvisa, 2010). Apresenta espectro de ação contra fungos, bactérias Gram-positivos, Gram-negativos e alguns tipos de vírus, principalmente quando está na forma de iodo livre (Moriya e Módena, 2008; Anvisa, 2010). Tem início de ação efetivada entre 1-2 minutos e efeito residual de 2-4 horas, no entanto sua atividade antimicrobiana pode ser inativada na presença de matéria orgânica como o sangue e também sofrer alteração por $\mathrm{pH}$, temperatura, tempo de exposição, concentração do iodo e presença de compostos inorgânicos como álcool e detergentes (Da Cunha et al., 2008). O PVPI a 10\% é mais indicado para a antissepsia do campo operatório de cirurgias oftalmológicas e otorrinolaringológicas (Anvisa, 2010; 2016).

O PVPI mantém as propriedades germicidas do iodo inalteradas apresentando algumas vantagens quando em soluções alcoólicas e aquosas desse agente, pois não queima a pele, não mancha os tecidos, diminui a probabilidade de reações alérgicas, não interfere no metabolismo e mantém a ação residual, apesar dessa ação diminuir na presença de matéria orgânica (Moriya e Módena, 2008).

\section{Considerações Finais}

A antissepsia do campo operatório em pequenos animais é um dos principais métodos de prevenção de infecção no pós-operatório, para isso as soluções antissépticas mais indicadas atualmente são o gluconato de clorexidina devido à sua rápida ação bactericida, fungicida e virucida mesmo na presença de fluidos corporais e poder residual prolongado, e o iodopovidona devido sua capacidade de inativar os micro-organismos em 12 minutos e ter efeito residual, apesar de sua ação diminuir na presença de matéria orgânica devido a presença do iodo.

\section{Conflito de interesse}

Os autores declaram não existir conflito de interesses.

\section{Referências}

Amato, A.C.M. História da cirurgia In: Morais, I.N. Tratado de clínica cirúrgica. Roca, 2006. p.3-17.

ANVISA - Agência Nacional de Vigilância Sanitária. Segurança do paciente: higienização das mãos. Brasília, 2010.

ANVISA - Agência Nacional de Vigilância Sanitária. Resolução da Diretoria Colegiada RDC $\mathbf{n}^{\circ} \mathbf{1 0 7}$, de 5 de setembro de 2016.

Arias, M.V.B.; Aiello, G.; Battaglia, L.A.; Freitas, J.C. Estudo da ocorrência de infecção hospitalar em cães e gatos em um centro cirúrgico veterinário universitário. Pesquisa Veterinária Brasileira, 33(6): 771-779, 2013.

Boerlin, P.; Eugster, S.; Gaschen, F; Straub, R.; Schawalder, P. Transmission of opportunistic pathogens in a veterinary teaching hospital. Veterinary Microbiology, 82(4): 347-359, 2001.

Brandão, G.Z.; Marçal, J.M.B.; Ramos, M.H.C.; Moreira, W.M.Q. A importância e eficácia da utilização de antissépticos nas mãos para redução de infecções hospitalares. Revista Fafibe On-Line, 8(1): 154-162, 2015.

Bhavan K.P.; Warren DK. Surgical preparation solutions and preoperative skin disinfection. Jounal of Hand Surgery, 34:940-941, 2009

Boucher, C.; Henton, M.M.; Becker, P.J.; Kirberger, R.M.; Hartman, M.J. Comparative efficacy of three antiseptics as surgical skin preparations in dogs. Veterinary Surgions, 110, 2018.

Burg, G.; Portela, O.; Paraginski, A.L.; Souza, V.; Silveira, D.D.; Horner, R. Estudo da eficácia de 
um novo produto à base de álcool gel utilizado na anti- sepsia em um serviço de nefrologia. Revista Medicina, 40(2): 2007.

Carraro, T.E. Os postulados de Nightingale e Semmelweis: poderlvital e prevençãolcontágio como estratégias para a evitabilidade das infecções. Revista Latinoamericana de Enfermagem, 12(4): 650-657, 2004.

Casey, A.L.; Lambert, P.A.; Elliott, T.S.J. Staphylococci. International Journal of Antimicrobial Agents, 29: 23-32, 2007.

Casey, A.L.; Itrakjv, A.; Birkett, C.; Clethro, A.; Bonser, R.; Graham, T.; Mascaro, J.; Pagano, D.; Rooney, S.; Wilson, I.; Nightingale, P.; Crosby, C.; Elliott, T. A comparison of the efficacy of $70 \% \mathrm{v} / \mathrm{v}$ isopropyl alcohol with either $0.5 \% \mathrm{w} / \mathrm{v}$ or $2 \% \mathrm{w} / \mathrm{v}$ chlorhexidine gluconate for skin preparation before harvest of the long saphenous vein used in coronary artery bypass grafting. American Journal of Infection Control, 43: 816-20, 2015.

Corsini, C.M.M.; Borges, A.P.B.; Alberto, D.S.; José, R.M.; Silva, C.H.O. Incidência de infecção do sítio cirúrgico e fatores de risco associados na clínica cirúrgica de pequenos animais. Arquivo Brasileiro de Medicina Veterinária e Zootecnia, 66(3): 737-744, 2014.

Couteur, P.L.; Burreson, J. Os botões de Napoleão: as 17 moléculas que mudaram a história. Rio de Janeiro: Zahar, 2009. p.89-102.

Da Cunha, M.L.; Procianoy, R.S.; Franceschini, D.T.; Cunha, M.L. Effect of the first bath with chlorheridine on skin colonization with Staphylococcus aureus in normal healthy term newborns. Scandinavian Journal of Infectious Diseases, 40(8): 615-620, 2008.

Dellinger, E.P. Infecções cirúrgicas e escolha dos antimicrobianos. In: Townsend, C.M. Tratado de cirurgia. Rio de Janeiro: Guanabara Koogan, 2003. p.182-200.

Dunning, D. Infeção da ferida cirúrgica e uso de antimicrobianos. In: Slatter, D. Manual de cirurgia de pequenos animais. São Paulo: Manole, 2007. p.113-122.

Felix, C.C.P.; Miyadahira, A.M.K. Avaliação da técnica de lavagem das mãos executada por alunos do curso de graduação de enfermagem. Revista da Escola de Enfermagem da USP, 43(1): 139-145, 2009.

Fernandes, A.T. Infecção hospitalar e suas interfaces na área da saúde. $1^{\mathrm{a}}$ ed. São Paulo: Atheneu, 2000. 1956 p.
Fernandes, A. T.; Ribeiro Filho, N.; Oliveira, A.C. Infecções do sitio cirúrgico. In: . Oliveira, A.C. Infecções hospitalares epidemiologia, prevenção e controle. Rio de Janeiro: Medsi, 2005. p. 93-123.

Fernando, F.S.; Silva, K.R.; Vignoto, V.K.C.; De Conti, J.B.; Pachaly, J.R.; Wosiacki, S.R. Avaliação microbiana de sítio cirúrgico relacionado ao tempo de procedimento e resistência a antimicrobianos em cães e gatos. Revista de Ciência Veterinária e Saúde Pública, 2(1): 26-33, 2015.

Fontana, R.T. As infecções hospitalares e a evolução histórica das infecções. Revista Brasileira de Enfermagem, 59(5): 703-706, 2006.

Francey, T.; Gaschen, F.; Nicolet, J.; Burnens, A.P. The role of Acinetobacter baumannii as a nosocomial pathogen for dogs and cats in na intensive care unit. Journal of Veterinary Intenal Medicine, 4(2): 177-183, 2008.

Garcia R. 2010. Surgical skin antisepsis. Perioperative Nursing Clinics, 5(4): 457-477.

Gonçalves, K.J.; Graziano, K.U.; Kawagoe, J.Y. Revisão sistemática sobre antissepsia cirúrgica das mãos com preparação alcoólica em comparação aos produtos tradicionais. Revista da Escola de Enfermagem da USP, 46(6): 1484-1493, 2012.

Goulart, D.R, Assis E.A, Souza M.T. Avaliação microbiológica da antissepsia pré-operatória das mãos. Revista de Cirurgia e Traumatologia Buco-maxilo-facial, 11(3): 2011.

Harari, J. Controle de infecção. In: Segredos em cirurgias de pequenos animais. $1^{\mathrm{a}}$ ed. Porto Alegre: Artmed, 2004. p. 25-32.

Levin, A.; Dias, M. Antimicrobianos: um guia de consulta rápida. $1^{\text {a }}$ ed. São Paulo: Atheneu, 2006. 188p.

Lister, J. Antiseptic principle in the practice of surgery. British Medical Journal, v. ii, p. 246, 1867.

Lister J. Effects of the antiseptic system of treatment upon the salubrity of a surgical hospital. The Lancet, 4-6, 1870.

Mangram, A.J.; Horan, T.C.; Pearson, M.L.; Silver, L.C.; Jarvis, W.R. Guideline for prevention of surgical site infection. American Journal of Infection Control, 27(2): 97-134, 1999.

Medeiros, L.K.L.; Rego, R.O.; Silva, M.M.; Henrique, F.V.; Felício Júnior, G.; Sousa, A.P.; Nóbrega Neto, P.I. Efeitos do banho prévio, da 
tricotomia e da antissepsia na redução da contaminação do sítio cirúrgico em cadelas submetidas à OSH eletiva. Pesquisa Veterinária Brasileira, 38(9): 1787-92, 2018.

Mcdonnell, G.; Russell, A.D. Antiseptics and Disinfectants: Activity, action, and resistance. Clinical Microbiology Reviews, 12(1): 14779, 1999.

McNeil, S.A.; Nordestrom-Lerner, L.; Malani, P.N.; Zervos, M.; Kauffman, C.A. Outbreak of sternal surgical site infections due to Pseudomonas aeruginosa traced to a scrub nurse with onychomycosis. Clinical Infectious Diseases, 33(33): 17-23, 2001.

Milstone, A.M.; Passaretti, C.L.; Perl, T.M. Chlorhexidine: expanding the armamentarium for infection control and prevention. Clinical Infectious Diseases, 46(2): 274-81, 2008.

Magalini S.; Pepe, G.; Panuzi, S.; De Gaetano, A.; Abatini, C.; Di Giorgio, A.; Foco, M.; Gui D. Observational study on preoperative surgical field disinfection: povidone-iodine and chlorhexidine-alcohol. Eurpean Review for Medical and Pharmacological Sciences, 17(24): 3367-3375, 2013.

Monteiro, V.L.C.; Coelho M.C.O.C., Mota R.A., Carrazzoni P.G., Tenório Filho F., Lima E.T.; Monteiro F.G.C. Anti-sepsia de pele de cães utilizandose clorhexidina a $2 \%$, povidine a $10 \%$ e álcool iodado a 5\%. Ciência Animal Brasileira, 11(1):7-12, 2001.

Moriya, T.; Módena, J.L.P. Assepsia e antissepsia: técnicas de esterilização. Medicina Ribeirão Preto, 41(3): 265-73, 2008.

Murray, P.R. Manual of clinical microbiology. $6^{\text {th }}$ ed. United States: ASM Press, 1995. 1482 p.

Orberg, C.; Schwartz, J.P.; Zander Grande, F.; Ruan, J.D.; Santos, E.B. Passagem de microrganismos através de luvas de procedimento e de luvas cirúrgicas antes de sua utilização. Revista de Odontologia da UNESP, 36(2): 127-130, 2007.

Pelosi, A. The operating room. In: Tobias, K.M., Johnston, S.A. Veterinary Surgery: Small Animal. Elsevier, 2017. p. 601-641.

Phillips, M.F.; Vasseur, P.B.; Gregory, C.R. Chlorhexidine diacetate versus povidone-iodine for preoperative preparation of the skin: a prospective randomized comparison in dogs and cats. Journal of the American Animal Hospital Association, 27: 105-108, 1991.

Poveda, V.B.; Galvão, C.M.; Hayashida, M. Análise dos fatores de risco relacionados à incidência de infecção do sítio cirúrgico e gastro-cirúrgicas. Revista da Escola de Enfermagem da USP, 37(1): 81-89, 2003.

Privitera, G.P.; Costa, A.L.; Brusaferro, S.; Chirletti, P.; Crosasso, P.; Massimetti, G.; Nespoli, U.M.; Petrosillo, N.; Pittiruti, H.; Scoppettuolo, L.; Tumietto, F.; Viale, P. Antisepsia da pele com clorexidina versus iodo para a prevenção de infecção do sítio cirúrgico: Uma revisão sistemática e meta-análise. American Journal of Infection Control, 45(2): 180-9, 2017.

Reis, L.M.; Rabello, B.R.; Ross, C.; Santos, L.M.R. Avaliação da atividade antimicrobiana de antissépticos e desinfetantes utilizados em um serviço público de saúde. Revista Brasileira de Enfermagem, 64(5): 2011.

Rezende, J.M. À sombra do plátano: crônicas de história da medicina. São Paulo: Unifesp, 2009. p. 181-200.

Rutkow, I. Joseph Lister and his 1876 tour of America. Annals of Surgery, 257(6): 11811187, 2013.

Santos, A.A.M.; Verotti, M.P.; Sanmartin, J.A.; Mesiano, E.R.A.B. Importância do Álcool no Controle de Infecções em Serviços de Saúde. Revista de Administração em Saúde, 4(16): 2002.

Santiago, A.R.; Charles Chamberland. Revista de la Sociedad Venezolana de Microbiologia, 25(1): 1-3, 2005.

Schulz, K.S. Princípios de Assepsia Cirúrgica. In: Fossum, T.W. Cirurgia de Pequenos Animais. $4^{\text {th }}$ ed. Rio de Janeiro: Mosby, 2014. p.01-10.

Seliskar, A.; Zdovc, I.; Zorko, B. Nosocomial Klebisiella oxytoca infection in two dogs. Slovenian Veterinary Research, 44(4): 115122, 2007.

Sievert, D.; Armol, R.; Halm, M.A. Chlorhexidine gluconate bathing: does it decrease hospitalacquired infections? Americam Journal of Critical Care. 20(2): 166-170, 2011.

Singh, A., Weese, J.S. Wound infections and antimicrobial use. In: _ Tobias, K.M., Johnston, S.A. Veterinary Surgery: Small Animal. $2^{\text {nd }}$ ed. 2017. p 530-549.

Stanley, D. Lights in the shadows: Florence Nightingale and others who made their mark. Contemporary Nurse, 24(1): 45, 2007.

Stehling, M.C.; Cunha, A.F.; Maria, E. Prevenção e controle de infecção em serviço de medicina veterinária. In: Martins, M.A. Manual de 
infecção hospitalar, epidemiologia, prevenção e controle. $2^{\text {nd }}$ ed. Belo Horizonte: Medice, 2001, p 915-927.

Viana, F.A.B. Abordagem da infecção em medicina veterinária: uso de antimicrobianos. In: _. Martins, M.A. Manual de infecção hospitalar: epidemiologia, prevenção e controle. 2. ed. Belo Horizonte: Medice, 2001, p.928-952.
Weese, J.S. A review of post-operative infections in veterinary orthopaedic surgery. Veterinary and comparative Orthopaedics and Traumatalogy, 21(2): 99-105, 2008.

WHO - World Health Organization. Guidelines on hand hygiene in health care: First Global patient safety challenge clean care is safer care. 2009. 